\title{
ANÁLISIS DEL POSICIONAMIENTO DEL FONDO MONETARIO INTERNACIONAL FRENTE A LA CRISIS DEL AÑO 2007
}

\author{
Ana Nora Feldman ${ }^{1}$
}

En el año 2008, la reina Isabel II de Inglaterra, al inaugurar el nuevo edificio de la London School of Economics, preguntó por qué nadie había predicho esa caída, cómo era posible que los expertos en economía no hubieran previsto la crisis. A lo cual el profesor Luis Garicano ${ }^{2}$ le respondió que "en cada etapa, alguien confiaba en otro alguien, y cada uno pensó que hacía lo correcto". La Reina tenía sus buenos motivos para desear conocer la respuesta pues se estima que, con la crisis, la familia Windsor perdió 50 millones de euros. ${ }^{3}$

El economista Gabriel Palma, en cambio, afirma que ningún economista ortodoxo pudo predecir la crisis financiera global porque "en los últimos 30 años su paradigma macroeconómico nos decía que solo existen agentes inteligentes, quienes solo pueden tomar decisiones óptimas ya que sus expectativas sobre el futuro las forman solo en forma racional." 4

Desde que comenzó la "Tercera Crisis del Capitalismo Contemporáneo" (Rapoport y Brenta, 2010b) mucho se ha

\footnotetext{
${ }^{1}$ Universidad Nacional Arturo Jauretche, Instituto de Ciencias Sociales y Administración. Email: anafeldman@gmail.com

${ }^{2}$ Profesor de Economía y Estrategias y Director del Grupo de Economía Empresarial y Estrategia, London School of Economics, Londres.

${ }^{3} \mathrm{http} / / /$ www.elblogsalmon.com/mercados-financieros/las-multiples-advertenciasjamas-escuchadas

${ }^{4}$ Gabriel Palma, http://ciperchile.cl/2013/11/12/por-que-la-economia-ortodoxatransfirio-su-obsesion-por-un-concepto-mercado-a-un-ritual-matematicas/
} 
discutido acerca de la incapacidad de previsión demostrada por el Fondo Monetario Internacional. La crisis iniciada en el año 2007 ocasionó la quiebra de importantísimas compañías dedicadas a préstamos hipotecarios. Recién tres años después el Fondo Monetario Internacional (FMI) reconoció la crisis.

Teniendo en cuenta el rol que, según su propio Estatuto, le corresponde al FMI, esta reacción tardía generó una áspera polémica entre economistas y académicos del desarrollo. No solo no pudo prever este tipo de situaciones, sino que, al impulsar la masiva salida de capitales y exigir la implementación de drásticos ajustes, el Fondo profundizó la recesión y agravó el impacto sobre los sectores menos favorecidos.

En este artículo se propone una aproximación no ortodoxa al estudio del Fondo Monetario Internacional, con la ayuda de técnicas estadísticas para el análisis textual (ADT) (Lebart y Salem, 1994). Se buscará desentrañar -a partir de los discursos de sus Directores- cuál ha sido el mensaje del FMI respecto de la crisis y los conceptos y lenguaje asociados. Assí como Pierre Bourdieu (Moscato y Wittwer, 1978) define el lenguaje como un indicador del sistema de creencias y actitudes o habitus lingüístico, William Labov ${ }^{5}$ nos enseña que el análisis de la lengua -y sus variacionesson un indicador de las "presiones sociales que se ejercen permanentemente" sobre esta.

Basado en una concepción ecológica para el tratamiento de datos cualitativos (Bolasco, 1993), el ADT permite procesar la información y obtener resultados sintéticos sin modificar la base de datos original (corpus), sea esta el producto de transcripciones (discursos, grupos o entrevistas), material de prensa u otros escritos, permitiendo la identificación de datos (conceptos, palabras, frases) de interés.

\footnotetext{
${ }^{5}$ W. Labov (http://www.ling.upenn.edu/ wlabov/) en su estudio en la isla Martha's Vineyard, citado en Moscato y Wittwer (1978)
} 
Existe hoy una gran cantidad de herramientas ${ }^{6}$ que permiten aplicar, de manera sencilla y rápida, técnicas de Análisis Estadístico de Datos Textuales (ADT) a diferentes tipos de textos, permitiendo el tratamiento de grandes volúmenes de datos a través de la identificación de palabras, segmentos o frases de interés mediante algunos parámetros de carácter cuantitativo (frecuencia, distribución estadística, etc.).

\section{Metodología}

El Análisis de Datos Textuales ${ }^{7}$ es un método inductivo para el análisis de textos, por el cual el lenguaje es considerado como un "sistema". El estudio del comportamiento de este sistema y de sus características se puede desarrollar a través del análisis de las unidades que constituyen el conjunto de valores propios. El estudio del lenguaje, como análisis del contenido, se realiza a través de un análisis de las distribuciones lexicales, o sea, con la ayuda de métodos estadísticos para el análisis de textos. A través de un enfoque cuantitativo, se puede efectuar un estudio de conjunto de las unidades lingüísticas que se encuentra dentro de un mismo texto.

En trabajos anteriores (Feldman, 2008) se ha demostrado que las técnicas de Análisis de Datos Textuales (ADT) utilizadas para la investigación social aplicada constituyen una herramienta útil en aquellos trabajos que requieren la exploración de datos no estructurados, o bien, la sistematización de información. Además de permitir construir un vocabulario general del corpus que se desea estudiar, un vocabulario específico para cada variable considerada en el análisis y un ranking de utilización de términos, el ADT ha permitido establecer, gracias al uso de técnicas multidimen-

\footnotetext{
${ }^{6} \mathrm{http}: / /$ lexicometrica.univ-paris3.fr/. Un listado de programas disponibles para el procesamiento se encuentra en https://fr.wikipedia.org/wiki/Analyse_de_ donn\%C3\%A9es_textuelles

${ }^{7}$ Fuentes Bolasco (2005), Lebart, L., Salem, A. y Bécue M. (2001) y Feldman, A. (1994, 1995)
} 
sionales, parámetros de comparación (por perfiles lexicales) y un espacio semántico para la visualización de conjunto.

El ADT brinda una "mejor resolución" en el estudio del contenido de un texto, dado que el análisis por conceptos es más flexible y cuidadoso que "un análisis por palabras clave o codificaciones" (Bolasco, 2005). Además, facilita al investigador incorporar la identificación y clasificación de las principales temáticas, a través del ranking de las formas textuales más frecuentes (Feldman, 2002).

El ADT permite determinar un vocabulario, identificar las formas más frecuentes y el vocabulario específico por segmento o grupo, en caso de que se busquen datos útiles para la comunicación, conocer los segmentos repetidos cuando la información debe ser sistematizada e identificar temáticas para el armado de dominios y las respuestas características por segmento. A su vez, las concordancias permiten contextualizar la información relevante identificada.

\section{El texto (y el contexto) de las comunicaciones del Fondo Monetario Internacional. Los discursos de los Directores}

El objetivo de este trabajo es reseñar de manera sintética el análisis que se ha realizado, desde el punto de vista discursivo, acerca de la posición del FMI respecto de la crisis económico-financiera mundial que se desató en el año 2007. Es decir, contempla una etapa que se inicia con "el derrumbe de los principales bancos de inversión así como de numerosas entidades financieras en EE.UU. y en Europa, donde hubo booms inmobiliarios similares y el sistema bancario estaba también profundamente involucrado en ese mercado y en operaciones dudosas" (Rapoport y Brenta, 2010a, p. 61). A partir de los discursos del FMI se buscará comprender el rol que se atribuyó el FMI y su concepción de la crisis a partir del análisis de los discursos de tres directores: Rodrigo De Rato (2004-2007), Dominique Strauss-Kahn (20072011) y Christine Lagarde (desde 2011). 


\section{Primeros resultados: el Corpus de los discursos de los Directores}

Una primera aproximación al texto la brinda el Vocabulario ${ }^{8}$ de los Directores (o sea la cantidad de palabras, u ocurrencias $-\mathrm{N}-$, encontradas en el texto) y la cantidad de formas (V) diferentes que se encuentran en él.

Tabla 1. Principales características lexicométricas del corpus DIRECTORES

\begin{tabular}{|l|c|c|c|}
\hline & $\begin{array}{c}\text { Cantidad de formas } \\
\text { (V) }\end{array}$ & $\begin{array}{c}\text { Ocurrencias } \\
\text { (N) }\end{array}$ & $\begin{array}{c}\text { Riqueza } \\
\text { vocabulario }\end{array}$ \\
\hline TOTAL CORPUS & 5942 & 42317 & $14 \%$ \\
\hline DE RATO & 3006 & 15387 & $20 \%$ \\
\hline LAGARDE & 3442 & 17445 & $20 \%$ \\
\hline STRAUSS-KAHN & 2461 & 9485 & $26 \%$ \\
\hline
\end{tabular}

Tratándose de un texto "hablado", aunque sus orígenes sean escritos, posee un 14\% de riqueza de vocabulario, 10 que denota una cierta amplitud en el lenguaje, así como de los temas de los cuales trata. ${ }^{9}$

Gráfico 1. Principales características lexicométricas de los discursos de cada director - corpus DIRECTORES

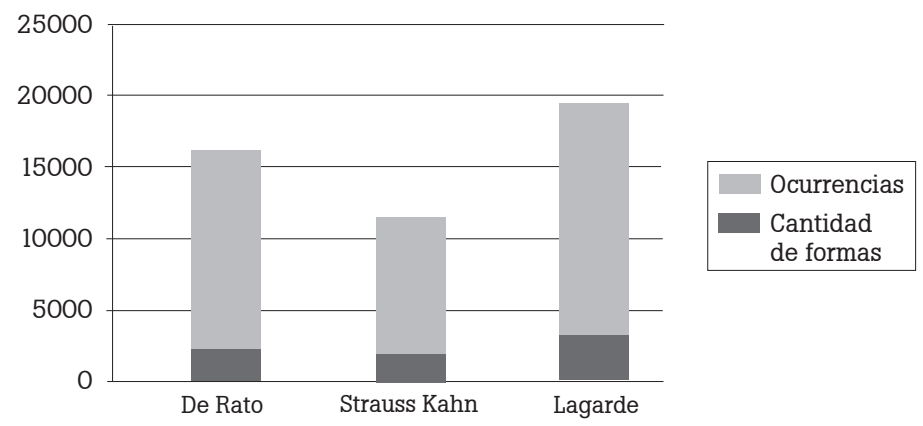

\footnotetext{
${ }^{8}$ Se llama vocabulario al conjunto de palabras diferentes. La cantidad de palabras diferentes en un texto define la amplitud del vocabulario (V). Siendo VI (número de palabras diferentes que aparecen i veces en un vocabulario).

${ }^{9}$ Normalmente estos textos originados en organismos internacionales poseen una riqueza que varia entre el 10 y el 12\%. Véase Feldman, 1994.
} 
Además de las lógicas diferencias lexicométricas entre los "hablantes", resulta de interés analizar la riqueza del vocabulario de cada uno de ellos (ver Tabla 1); se puede inferir algo acerca del tipo de discurso y también eventuales similitudes/diferencias entre los directores. De Rato y Lagarde poseen un lenguaje menos profuso. Muchas palabras, pero todas bastante similares. En cuanto al director Strauss Kahn, a pesar de incorporar al análisis solamente tres discursos, demuestra maestría de un amplio lenguaje. Sin embargo, no siempre es cierto que esto signifique mayor dominio sobre el tema; en el caso que se analizó en su momento acerca del lenguaje del Programa de Naciones Unidas para el Desarrollo (PNUD) (Feldman, 1994) el aumento de la cantidad de palabras diferentes no indicaba mejor manejo de la temática sino exactamente lo contrario.

Al tratarse de diferentes períodos de análisis y cantidad de discursos no equidistribuidos se trabajará sobre la "cantidad de formas" (V), es decir, el vocabulario que utiliza cada uno de los tres directores en análisis. Más allá de una lógica mención a su propia institución, existen algunas palabras que son interesantes en cuanto a la descripción que nos hacen sobre la institución "hablante".

Por ejemplo, puede resultar de interés conocer cuáles son las instituciones mencionadas por los Directores. Sin embargo, resulta decepcionante advertir que el Fondo Monetario Internacional habla... ide sí mismo! De manera marginal nombra al Banco Mundial y al G20. Esta autorreferencialidad ya ha sido trabajada para el caso ya mencionado del PNUD (Feldman, 1995). Generalmente, estos organismos supranacionales tienen como principal objetivo -no declarado- la propia supervivencia. Para esto deben fundamentar esa necesidad a partir de la reafirmación de una identidad propia, que les brinda idea de autogeneración y legitimación. Sus textos, sus discursos, sus trabajos estarán todos orientados, además de al objetivo por el cual fueron creados (como en el caso del FMI, el de regular los flujos fi- 
nancieros y los préstamos de última instancia para los países), a perpetuarse en el tiempo.

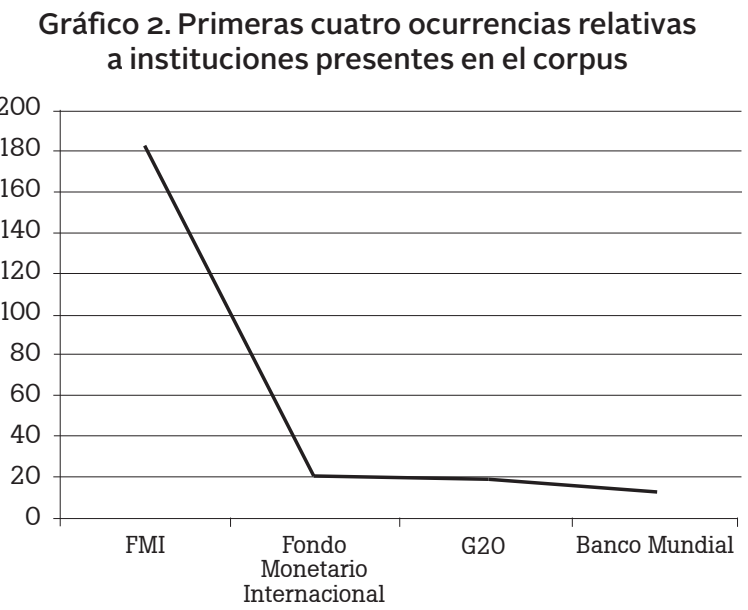

El FMI es un organismo multilateral y multinacional, pero que no presta atención a todos por igual ¿Qué países son mencionados por los Directores? Más allá de la referencia al mundo, los países asiáticos parecerían ser eje de un espacio de atención. El 23\% de las menciones a áreas geográficas hacen referencia a Asia, seguido por Estados Unidos y Europa. Ni África ni América Latina ameritan mucha atención.

Gráfico 3. Distribución geográfica de los temas de interés mencionados por los Directores
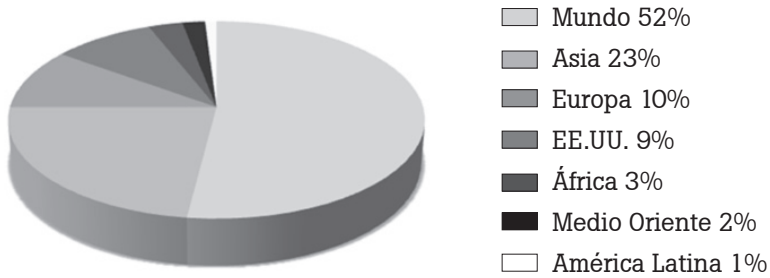


\section{Lenguajes peculiares o específicos}

La estadística de datos textuales permite analizar estos discursos de manera comparativa. Se puede prescindir de la lógica simplemente numérica: más palabras -más conceptos- más contenido y, sobre todo, brinda un dato de tipo significativo: más allá de cuánto hablen, lo que importa es qué palabras utilizan de manera diferente del resto de los hablantes.

Según una ley que se aplica a la lingüística sabemos que toda lengua se distribuye como una curva hipergeométrica (la Ley de Zipf ${ }^{10}$ ). A partir de esta herramienta se analizarán las "peculiaridades" del discurso de cada uno de los directores. La idea es poder reflejar, de manera empírica, su idea, su concepción del mundo, así como el eje de los discursos pronunciados, más allá del momento de su pronunciamiento.

Se analizarán, en primer lugar, aquellas palabras con mayor nivel de significatividad estadística que, además, son exclusivas del hablante. Esto permitirá establecer el estado de situación particular en el que se emite el mensaje y el interés exclusivo del hablante.

Tabla 2: Los discursos de los Directores ${ }^{11}$

\begin{tabular}{|c|c|c|c|}
\hline & $\begin{array}{c}\text { Palabras } \\
\text { Exclusivas* }\end{array}$ & Temáticas de interés & Acciones asociadas \\
\hline & & \multicolumn{2}{|c|}{ (Formas características**) } \\
\hline De Rato & $\begin{array}{l}\text { PETRÓLEO, } \\
\text { SOLIDEZ, } \\
\text { EMPRESAS, } \\
\text { SUBVENCIONES }\end{array}$ & $\begin{array}{l}\text { MERCADOS FINANCIEROS, PETRÓLEO, } \\
\text { MACROECONOMÍA, CRÉDITO, ACTI- } \\
\text { VOS, AHORRO, PRECIOS, RIESGO/S, } \\
\text { INSTRUMENTOS, ECONOMÍAS, BANCOS } \\
\text { CENTRALES, COMERCIO, INVERSIÓN, } \\
\text { MERCADO, GLOBALIZACIÓN }\end{array}$ & $\begin{array}{l}\text { DESARROLLO, } \\
\text { DESEQUILIBRIOS, } \\
\text { ENVEJECIMIENTO, } \\
\text { SUBVENCIONES, } \\
\text { SOLIDEZ, LIBERALI- } \\
\text { ZACIÓN, CAMBIOS }\end{array}$ \\
\hline
\end{tabular}

${ }^{10}$ Una lengua posee una cierta cantidad limitada de palabras que se repiten muchas veces y muchas otras (la inmensa mayoria) que se repiten pocas veces en un texto (Lebart y Salem, 1994).

${ }^{11}$ Las palabras en MAYÚSCULAS son aquellas que se encuentran efectivamente en los textos analizados. 


\begin{tabular}{|c|c|c|c|}
\hline $\begin{array}{l}\text { Strauss- } \\
\text { Kahn }\end{array}$ & $\begin{array}{l}\text { UNIÓN, } \\
\text { INCLUSIÓN / } \\
\text { INCLUSIVO, } \\
\text { IDEA, MUJER, IN- } \\
\text { TERCONECTADO, } \\
\text { CORTAFUEGOS, } \\
\text { DESIGUALDAD, } \\
\text { TOKIO }\end{array}$ & $\begin{array}{l}\text { ASIA, MALASIA, CUENTAS, FISCAL, } \\
\text { GENERACIÓN (MUNDO, ZONA, } \\
\text { REGIONES), EMPLEO, INCLUSIÓN/ } \\
\text { INCLUSIVO, MUJER, EUROPA, DEUDA, } \\
\text { DESIGUALDAD }\end{array}$ & $\begin{array}{l}\text { CUMPLIR, UNIÓN, } \\
\text { INTEGRACIÓN, } \\
\text { HISTORIA, } \\
\text { CORTAFUEGOS, } \\
\text { COOPERACIÓN, } \\
\text { FORTALECER, } \\
\text { AYUDAR }\end{array}$ \\
\hline Lagarde & CONFLICTO & $\begin{array}{l}\text { PAÍSES DE BAJO INGRESO, PAZ, } \\
\text { PRÉSTAMOS, G 20, FINANCIAMIENTO, } \\
\text { LÍDERES, RECURSOS, CRISIS, } \\
\text { GUERRA, CONFLICTO, INTERNACIONAL, } \\
\text { SOCIAL, ECONÓMICO, FMI, RESERVAS, } \\
\text { PRÉSTAMO, ECONÓMICA, POBRES }\end{array}$ & $\begin{array}{l}\text { PROGRAMAS, } \\
\text { CONDICIONES, } \\
\text { NECESIDADES, } \\
\text { RESPALDO, ESTÍMULO, } \\
\text { POLÍTICAS, } \\
\text { PROSPERIDAD, AYUDA, } \\
\text { COOPERACIÓN }\end{array}$ \\
\hline
\end{tabular}

* Solo aparecen en el discurso de un Director.

** Estadísticamente significativas

\section{El discurso del director Rodrigo De Rato}

Con el foco en las EMPRESAS, este Director hablará sobre todo de aspectos financieros relacionados con el nuevo panorama para las multinacionales, que encontraron oportunidades ventajosas, para "diversificar sus actividades en los países en los que las realizaban, disponiendo de una mayor capacidad para gestionar el riesgo de sus operaciones" $(\mathrm{Pa}-$ lazuelos, 1998 ). Estas volcaron la liquidez en las empresas, en lugar de utilizarla con fines productivos, para la compra de instrumentos financieros portadores de interés o rendidores de dividendos (Magdoff y Sweezy, 1998). Asimismo, "las inversiones de los fondos de inversión en acciones de empresas los convirtieron en los grandes accionistas de las corporaciones" (Sevares, 2009). Fusiones y adquisiciones estaban a la orden del día, habiendo llegado en el año 2007 a 1,6 billones de dólares.

Al utilizar la palabra ACTIVOS, De Rato está refiriéndose al aumento de los activos financieros globales que, desde los años ochenta, ha tenido una relación peligrosa con el PIB mundial. Pasaron de representar el 20\% superior al PIB a, en 2007, tener un valor 3,5 veces más elevado que el PIB. En cuanto al AHORRO, este tema representó un problema importante en el período en que De Rato estuvo al fren- 
te del FMI. En Estados Unidos el ahorro de las familias cayó del 11\% del PIB en 1983 al 3\% en 2007.

Las recomendaciones de política económica efectuadas por este Director Gerente son de carácter amplio, genéricas y no implican una coherencia específica destacable. Así, resulta factible encontrar en su discurso términos que pueden, inclusive, representar políticas económicas concretas que sean disímiles o contrapuestas entre sí (como, por ejemplo, promover contemporáneamente la liberalización y las subvenciones a los agentes económicos).

\section{El discurso del director Strauss-Kahn}

Este Director, además de tener un currículo sustancialmente diferente de sus otros dos colegas, hace uso de un vocabulario y se refiere a temáticas muy distantes de las utilizadas tanto por su antecesor, el director De Rato, cuanto por su sucesora, la directora Lagarde. Llama la atención que, si bien en los informes del FMI se mencionan corrientemente los paraísos fiscales como "vehículo de inversión financiera" a través de entidades que "se utilizan con frecuencia para el lavado de dinero y la evasión tributaria," ${ }^{12}$ Strauss-Kahn se concentra, en cambio, en temáticas relacionadas con la desigualdad, la redistribución, la inclusión y la exclusión.

Hay, además, una palabra que, si bien no es exclusiva, es de altísima frecuencia en el discurso y es el CONTAGIO. Refiriéndose sobre todo a lo que fue una de las principales preocupaciones del período en el que Strauss-Kahn fue director del Fondo. La "fiebre inmobiliaria" y su consecuente burbuja, que tuvo sus comienzos en Estados Unidos, se difundió rápidamente a otros lugares del mundo, como es el caso de España. La caída del mercado de hipotecas subprime se extendió rápidamente y tuvo un efecto dominó que no fue captado ni corregido a tiempo.

\footnotetext{
${ }^{12}$ Señalado por Vito Tanzi (jefe de impuestos del FMI) en "La globalización y la acción de las termitas fiscales", en Finanzas y Desarrollo, $n^{\circ} 38$, marzo de 2001. Citado en Sevares, Julio, op. cit.
} 
"La crisis que comenzó como un problema financiero se trasladó rápidamente al sector real. A fines de 2008, las economías industrializadas entraron en diferentes grados de recesión y los organismos internacionales comenzaron a revisar a la baja en forma casi semanal sus previsiones de crecimiento para ese año y para 2009" (Sevares, 2009, p. 95)

El conjunto de proposiciones de política económica propuestas por este Director Gerente resulta la más coherente de las tres aquí analizadas. Se destaca que Strauss-Kahn promueve la integración, la unión (bancaria y/o financiera) y la cooperación, términos que implican la promoción de un escenario en el cual las decisiones globales de política económica privilegien la adopción de consensos que sean, necesariamente, generados en un ámbito de colaboración internacional y/o multilateral. Esto último también es plenamente consistente con el rol que usualmente asume el FMI en relación con el establecimiento de la agenda económica internacional.

Sin embargo, teniendo en cuenta que durante el período en que Strauss-Kahn fue el Director del Fondo Monetario la crisis tomó estado público y se produjeron los principales contagios a nivel mundial, llama la atención que estas características y la peligrosidad de la crisis desatada no se registran en el lenguaje utilizado por el Director.

\section{El discurso de Christine Lagarde}

Las medidas de política económica asociadas con la actual Directora Gerente del FMI están inmersas en el ámbito de un escenario de crisis económica internacional de alto impacto. Así, la funcionaria destaca la necesidad de aplicar programas y acciones de respaldo o estímulo a la actividad económica, así como también acciones de ayuda y cooperación. Asimismo, es la única funcionaria que destaca el término "conflicto", lo cual puede estar relacionado con el crítico escenario internacional descrito inicialmente. 
Así las cosas, la Directora Lagarde pone el foco en las cuestiones relacionadas con las consecuencias de la/s crisis económica/s más que en el análisis acerca de cuáles son los aspectos salientes de las crisis, qué provocó esas crisis y qué debería hacerse para limitarlas o extinguirlas.

Es la misma Directora quien ayuda a interpretar sus discursos cuando afirma que "La institución recomienda algunas soluciones que son "dolorosas" pero cuyos resultados deben ser evaluados en el largo plazo." ${ }^{13}$ Cabe señalar, además, que ni los desequilibrios ni la estabilidad ni las cuestiones financieras hacen parte del discurso de Lagarde.

El deterioro de las carteras de préstamos de varios bancos y la imposibilidad de los gobiernos de detener esto desencadenó una espiral en la que: "varios bancos se endeudaron para prestar más que sus depósitos". Los bancos buscaron reforzar su capital, "pero los inversores ya no quisieron prestarles. Y los depositantes, temiendo que los bancos quebraran, trasladaron su dinero a bancos en países más fuertes, como Alemania", señala McClain.. ${ }^{14}$

Lagarde pone voz a una institución que propone soluciones dolorosas que no admiten la discusión o la negociación.

\section{Semejanzas y diferencias de los Directores del período analizado}

Con este primer acercamiento a los discursos a partir de las distribuciones estadísticas de las palabras utilizadas verificamos que en el discurso de los Directores no se afronta directamente lo que la crisis significa. Strauss-Kahn demuestra maestría en el uso de un amplio lenguaje, pero esto no necesariamente indica dominio del tema que se está desarrollando. La motivación de esta particularidad puede estar basada en la explicación de conceptos que son poco cono-

${ }^{13} \mathrm{http}$ ://eleconomista.com.mx/economia-global/2013/06/27/papel-fmi-serareconocido-algun-dia-lagarde

${ }^{14}$ Understanding the European Crisis Now, Dylan Loeb McClain. http://www. nytimes.com/interactive/2012/06/14/business/global/understanding-theeuropean-crisis.html?_r=0 
cidos por los lectores/oyentes o bien que se habla de tópicos sobre los cuales no existe un amplio consenso. Este último fue el caso de las variaciones en el lenguaje, la longitud y la riqueza del vocabulario que el Programa de Naciones Unidas para el Desarrollo utilizó para la instalación y defensa del que entonces era un nuevo concepto, es decir, el de Desarrollo Humano (Feldman, 1994).

Las recomendaciones de política económica efectuadas por el doctor Rodrigo De Rato son de carácter amplio, genéricas y no implican una coherencia especifica destacable. Así, resulta factible encontrar en su discurso términos que pueden, inclusive, representar políticas económicas concretas que sean disímiles o contrapuestas entre sí (como, por ejemplo, promover contemporáneamente la liberalización y las subvenciones a los agentes económicos). Es el único que pone el foco en materias primas y variables básicas de la economía, además de describir un mundo en búsqueda de equilibrio y desarrollo.

Dominique Strauss-Kahn era el Director Gerente del FMI cuando se desencadenó la peor crisis económica que el mundo registra en su memoria después de la Gran Depresión. Casi como si hubiera buscado que el foco se pusiera en otro lugar, este Director de lenguaje florido se concentra en temáticas relacionadas con la desigualdad, la redistribución, la inclusión y la exclusión. Todos estos temas que no forman parte del "folclore" del Fondo Monetario.

El conjunto de proposiciones de política económica de este Director Gerente es el más coherente de los tres. Strauss-Kahn promueve la integración, la unión (bancaria y/o financiera) y la cooperación, términos que implican la promoción de un escenario en el cual las decisiones globales de política económica privilegien la adopción de consensos que sean, necesariamente, generados en un ámbito de colaboración internacional y/o multilateral. Esto último también es plenamente consistente con el rol que usualmente asume el FMI en relación con el establecimiento de la agenda económica internacional. Sin embargo, teniendo 
en cuenta que, durante el periodo en que Strauss-Kahn fue el Director del Fondo Monetario, la crisis tomó estado público y se produjeron los principales contagios a nivel mundial, llama la atención que estas características y la peligrosidad de la crisis desatada no se registre en el lenguaje utilizado por este. La autonomía del personal técnico del FMI podría estar en la base de esta "disonancia conceptual".

Sumergido en una crisis institucional, el Fondo busca, sobre todo en el período de este Director Gerente, cambiar su imagen y su accionar procurando la óptica sugerida por Stiglitz en su libro del año 2002, El Malestar en la Globalización, es decir, mirando más al mundo en desarrollo y buscando por allí una estrategia adecuada para evitar la profundización de la crisis.

El nombramiento de Strauss-Kahn parece haber sido la estrategia más adecuada de los países del G7 en la búsqueda de una relegitimación del organismo para que este volviera a ser protagonista en el escenario internacional. Un hombre culto, formado en la academia, más de izquierda que de derecha (precandidato socialista en Francia), su incorporación cambió de manera notable el discurso del Fondo Monetario al prestar atención a temáticas hasta el momento no relevantes, como la desigualdad y la distribución de la riqueza.

Strauss-Kahn logró darle visibilidad al organismo hablando desde otro lugar muy diferente al lenguaje y las temáticas de Rodrigo De Rato, su antecesor. Por supuesto, no se está hablando de una ruptura del statu quo del organismo, ya que Strauss-Kahn siempre fue funcional al establishment.

Es en un contexto mucho más crítico y con amplias discusiones sobre el rol del Fondo que se inserta el discurso de Christine Lagarde, quien recurre a la amenaza del conflicto, dibujando un panorama en el que no hay lugar para la negociación ni para la búsqueda de consenso. Este pasaje de la disuasión a la acción en el discurso de los organismos internacionales se relaciona con la búsqueda de validación 
del rol de la institución, como ya se estudió en trabajos anteriores. ${ }^{15}$

Las medidas de política económica mencionadas por Lagarde son las de un escenario de crisis económica internacional de alto impacto. La aplicación de los programas impuestos por el Fondo es de suma importancia para lograr salir de la crisis. Lagarde habla desde un FMI pesimista, prescriptor y normativo que actúa en situación de crisis. Una institución que propone soluciones dolorosas que no admiten la discusión o la negociación.

\section{Conclusiones}

Con la ayuda del ADT se ha podido conocer, desde el punto de vista del análisis del contenido, la posición del Fondo Monetario Internacional ante la crisis financiera internacional de mayor envergadura después de la Gran Recesión de 1930. Un aspecto que se deseaba conocer era si el FMI había podido comprender, con la suficiente rapidez, la lectura de los indicadores económicos que alertaban sobre una situación de desequilibrio y fragilidad.

La lectura de los discursos en general ha permitido comprender cuál fue el rol que se atribuyó el organismo y quiénes eran los protagonistas de esta crisis, desde su punto de vista.

Sabemos también que las intervenciones del FMI están directamente relacionadas con la aplicación no neutral de criterios técnicos y políticos y que no responden al acuerdo de la llamada comunidad internacional sino a la que podría llamarse comunidad internacional de peso real. ${ }^{16}$

Debemos recordar que las motivaciones de los discursos y los tópicos que mencionan los Directores están directamente relacionados con el concepto relativo a cómo de-

\footnotetext{
${ }^{15}$ Se hace referencia al pasaje del concepto de "desarrollo humano" al de "seguridad humana" para el PNUD en el año 1994 (Feldman, 1994).

${ }^{16}$ Con esta expresión se hace referencia a lo que hoy se ha dado en llamar comúnmente la Troika (Banco Central Europeo, Unión Europea y Fondo Monetario Internacional) y a la Reserva Federal de los Estados Unidos.
} 
be leerse la situación económica mundial y el rol del Fondo Monetario en dicho contexto, en acuerdo con los intereses económicos y políticos de los países centrales.

Ni previsión ni visión han caracterizado los discursos de estos Directores; tampoco podría afirmarse que hay análisis de la crisis que se inició en el año 2007.

En los discursos analizados, Rodrigo De Rato (15.387 ocurrencias) y Christine Lagarde (17.445 ocurrencias) desarrollan un discurso extenso con una variedad de formas (3.006 el primero y 3.442 la segunda) y con una riqueza media de vocabulario (20\%). En el caso de Dominique Strauss-Kahn, sus discursos son mas breves (9.485 ocurrencias) pero con una riqueza mayor (26\%) correspondiente a 2.461 palabras diferentes. Strauss-Kahn demuestra maestría en el uso de un amplio lenguaje, pero esto no necesariamente indica dominio del tema que se esta desarrollando.

Ya se ha demostrado que las propuestas del doctor Rodrigo De Rato son amplias y genéricas y no muy coherentes. Dominique Strauss-Kahn, en su afán por reposicionar al Fondo, privilegia la búsqueda de consensos con una mirada especial hacia lo social. La estrategia representada por Strauss-Kahn fue abandonada por su sucesora Christine Lagarde, quien no busca consenso sino amedrentar con la amenaza del conflicto.

Mucho se ha discutido acerca de la capacidad de previsión de la crisis y cuál fue la posición del Fondo Monetario respecto de sus consecuencias. En este artículo se ha analizado el discurso de los Directores del FMI utilizando, como herramienta para la selección de palabras y contextos, los resultados de la aplicación de técnicas estadísticas de análisis estadístico de datos textuales.

La negación de la crisis, solo reconocida en el año 2010, es coherente con la misión que el FMI considera que debe cumplir, aleccionando sobre la forma en que los gobiernos deben corregir sus economías (Weisbrot, Cordero y Sandoval, 2009). Todo esto ignorando que "sus recetas fallaron" 
(Stiglitz, 2002, p.20) pues "las políticas de ajuste estructural [...] produjeron hambre y disturbios" beneficiando a quienes poseían más recursos mientras que "los pobres en ocasiones se hundían aún más en la miseria". Un mensaje, un emisor, un objeto y una misión que ha fallado, pues el FMI no ha cumplido con su rol de evitar que el mundo caiga nuevamente en una nueva Gran Depresión.

Con Joseph Stiglitz (2002, p. 64) compartimos la idea de que "ha llegado el momento de cambiar algunas de las reglas del orden económico internacional" y de "repensar cómo se toman las decisiones a nivel internacional". Con este y otros trabajos de análisis del Fondo se desea aportar en esa dirección.

\section{Referencias}

Bolasco, Sergio (1993) "Choix de lemmatisation en vue des reconstructions syntagmatiques du texte par l'analyse des correspondences". Secondes Journées Internationales d'Analyse Statitique des Données Textuelles. Montpellier, Octubre 21-22.

Bolasco, Sergio (2005) "Statistica Testuale e Text Mining: alcuni paradigmi applicativi". Quaderni di Statistica, Vol.7.

Feldman, Ana (1994) "Il Concetto di Sviluppo Umano secondo le Nazioni Unite: Analisi del Contenuto", Tesis, Facoltà di Scienze Statistiche, Demografiche ed Attuariali, Università di Roma La Sapienza.

Feldman, Ana (1995), "Il concetto di sviluppo umano secondo le Nazioni Unite: analisi del contenuto". In Bolasco, Sergio, Ludovic Lebart, André Salem (eds). JADT 1995 - Analisi statistica dei dati testuali: CISU, 2 voll.

Feldman, Ana (2002) "La integración del Análisis Textual a los estudios de Calidad de Servicio y Satisfacción del Cliente", en A. Morin \& P. Sébillot (eds.), Gèmes Journées internationales d'Analyse statistique des Données Textuelles, volumen 1, Rennes: Institut National de Recherche en Informatique et en Automatique. 
Feldman, Ana (2008) "Uso de Técnicas de Análisis de Datos Textuales en la Investigación Social Aplicada". Tesis de Maestría, Universidad Nacional de Tres de Febrero y Università di Bologna (Buenos Aires).

Lebart, Ludovic y André Salem (1994) Statistique Textuelle, Paris: Dunod.

Lebart, Ludovic, André Salem, y Mónica Becue (2001) Análisis Estadístico de Textos, Leida: Milenio Editorial.

Magdoff, Harry y Paul Marlor Sweezy (1998) Estancamiento y explosión financiera en Estados Unidos. Buenos Aires: Siglo XXI Editores.

Moscato, Michel y Jacques Wittwer (1978) La Psychologie du Langage, Collection Que sais-je?, Paris: Presses Universitaires de France.

Palazuelos Enrique (1998) La globalización financiera, Madrid: Editorial Síntesis.

Rapoport Mario y Noemi Brenta (2010a) Las grandes crisis del capitalismo contemporáneo. Buenos Aires: Capital Intelectual.

Rapoport Mario y Noemí Brenta (2010b) La crisis económica mundial: ¿el desenlace de cuarenta años de inestabilidad?, Revista Problemas del Desarrollo, 163 (41).

Sevares, Julio (2009) El estallido de la Superburbuja I. Causas de la Crisis Económica. Buenos Aires: Capital Intelectual.

Stiglitz, Joseph (2002) Globalization and Its Discontents, Stiglitz, J.E., W.W. New York: Norton \& Company.

Weisbrot, Mark, José Cordero, y Luis Sandoval (2009) Empowering the IMF: Should Reform be a Requirement for Increasing the Fund's Resources? Washington, D.C: Center for Economic and Policy Research. 